\title{
Critical Response: Investments in the imaginary: Commercial drone speculations and relations
}

\section{Those Mystery Drone Swarms!!! \\ Pre-Submission Draft - Dr Paul Cureton, Lancaster University.}

Many future visions present a 'frictionless future' for the built environment one of technological optimism through the smart city, which often neglects key social relations and dynamics of urban spaces (Dunn and Cureton 2019). In this case, promotional videos and design patents for Amazon Air are investigated as speculative artefacts, which 'capture the future'. The mapping of the techno-political 'dronescape' by Anna Jackman and Maximilian Jablonowski analyse media representations, which function as key speculative tools. The media discussed reveals absences within the visions. The results reveal the embodied ideas in the patents and advertisements of the various mega-corporates with R\&D capability. There is much robotic innovation but at the same time there is little understanding of integration within the wider built environment and this reflects in Prime Air's 'vision videos' as carefully analysed by the authors. Drones are seemingly integrated and seemingly expanded as services in often-linear modes of futuring. In addition, there are few studies on how 'drones futures' project in the mobility turn (Jensen 2020). Drone technology is part of a system of technological stacking and fusing of process and components and human application in civilian settings. For example, it would take satellite technology and GPS establishment to provide a system of position and manoeuvre for modern civilian drones.

Drone futures and the mode of speculation often rest on the strategies, processes and deployment from military applications and as the authors' state is "reimagined from their battlefield positions". While this crossover has many benefits including rigorous testing regimes, and safety cultures for aviation these modes of integration do not account for irrational, dystopian and negative reception of the systems. Fears exacerbate through the reception of new technology. In one such example, various fears emerged regarding the purpose of drone missions in various drone swarm night sightings from January 2020 flying in Colorado, Nebraska and Wyoming in the USA. Sightings of a drone swarm at night executing grid manoeuvres perpetuated further false reports and intense speculation of the culprits. Various speculative articles appeared; the power of the mystery in full effect with one article authored by Jeff Wise indicating the possibility of Wing a subsidiary of Alphabet. Some of the 'vision videos' discussed in the article of Amazon air and their world-building exercise and integration into everyday life can also be misunderstood as now capability, as in this case of Wing (allegedly) and the night sightings, rather than a futurological possibility. These vision videos provide as the article states, 'realism'. It is through world-building that fiction and reality are blurred exacerbating fears and mystery of the drone.

As Jackman and Jablonowski carefully unpack the design patent of Amazon's drone mobility system, the analysis develops the contribution to wider 'drone imaginaries' and infrastructural blueprint. This blueprint of aerial mobility and service emerges as a wider trope in histories of the future as we have seen in Archigram's Giant Skyhook dirigible in the 
temporality of Instant City (1970) which very much resonates with Amazon's Blimp drone hive patent. A video clip released in Hiroshima, Japan, April 2019 showed an Amazon blimp, based on the Lockheed Martin P-791 hybrid prototype, which matched its submitted patents for airborne fulfilment centres. The video clip led to various speculations in social media and news centres believing that the media demonstrated secret trials, when in fact was the work of a CGI artist called Zozi. The hybrid airship can transport much larger payloads than quadcopter and requires very little docking infrastructure, making the airship ideal for access to remote locations. With the current prototype able to hold up to 21,000 kg/47,000 lbs, 19 passengers and a range of $1400 \mathrm{~nm}$ (Nautical Mile) this prototype fused with Amazon Air in the video leading to a convincing media form (Cureton 2020: 111-119). Indeed, in this case, two complementary techniques developed by Coulton and Lindley seem appropriate for pathways in this speculative media creation for alternative drone imaginaries which utilise 'vision video' techniques and subvert this media for new diverse and alternative potentials. The first is speculative ontography, which allows the creation of constellation diagrams of the independent and interdependent relationships and perspectives in potential more-than-human ecologies (Lindley et.al, 2020) and Design Fiction (Coulton et.al 2017) as means of bringing these constellations to life for discussion with various potential stakeholders.

These Amazon Air visions as the author's state, one of the loaded everydayness of drone logistics combined with 'innovation' branding and convenience promote a monopoly on the volumetric airspace reserved for All UAS operations in future national policies. The articles identification of the 'realness' and lifeworld building is an effective device for the "entrenching of techno-fetishist neoliberal agenda positing technology as a privileged and panacea agent of futurity". Amazon Air et al promote mastery of endemic problems in cities themselves and offer critiques of planning whilst at the same time promoting ideas of digital connectedness and consumerism through drone delivery and dirigible travel. These circulatory systems are innovations driven by the failure of other transportation and infrastructure modes while also providing a degree of 'smartness' to which many cities seek. However, how much does the patent have to address social relations and urban fabric? In some way, the diagrammatic evasiveness is deliberate to avoid the issue and complexity of physical space. The design patent is devised because its dual nature is one that presents a seemingly fluid schematic yet at the same time deliberately obscures the complexity of everyday life. Combined with the aforementioned 'vision' videos means that critical integrations such as this are fundamental. What the media does provide is a template and method for alternative design fictions and alternative social-cultural imaginaries to which drones can be understood through speculation and deployed in beneficial ways. Drone Futures and modes of speculation are critical dialogues for our (aerial) infrastructures but also key debates for our relationship with new technologies. Alternative speculations would develop new territories of understanding, rather than fear in the unknown, the 'mysteries of drones', fears, irrationalities and captured 'futures' of mega-corporates. For this, I thank Anna Jackman and Maximilian Jablonowski for this analysis and investigation opening up an important area of enquiry. 


\section{Bibliography}

Cureton, P. (2020) Drone Futures: UAS for Landscape \& Urban Design. (London, Routledge).

Coulton, P, Lindley, JG, Sturdee, M \& Stead, M. 2017, Design fiction as world building. in Proceedings of Research through Design Conference 2017. Research through Design (RTD) conference 2017, Edinburgh, United Kingdom, 22/03/17.

Dunn, N. and Cureton, P. (2019) Frictionless Futures: the Vision of Smartness and the Occlusion of Alternatives. In Figueiredo, Krishnamurthy and Schröder (eds) Architecture and the Smart City. (New York, London, Routledge).

Jensen, O.B. (2020) Thinking with the drone - visual lessons in aerial and volumetric thinking, Visual Studies. https://doi.org/10.1080/1472586X.2020.1840085.

Kaplan, C. (2020) Atmospheric politics: protest drones and the ambiguity of airspace. Digital War. https://doi.org/10.1057/s42984-020-00005-y.

Lindley, J, Akmal, H \& Coulton, P. (2020) 'Design Research and Object-Oriented Ontology', Open Philosophy, vol. 3, no. 1, pp. 11-41. https://doi.org/10.1515/opphil-2020-0002

Wise, J. Who's Behind Those Mystery Drone Swarms? An Investigation, Jan. 11, 2020 https://nymag.com/intelligencer/2020/01/whos-behind-those-mystery-drone-swarms-aninvestigation.html. 